
DESY $74 / 32$
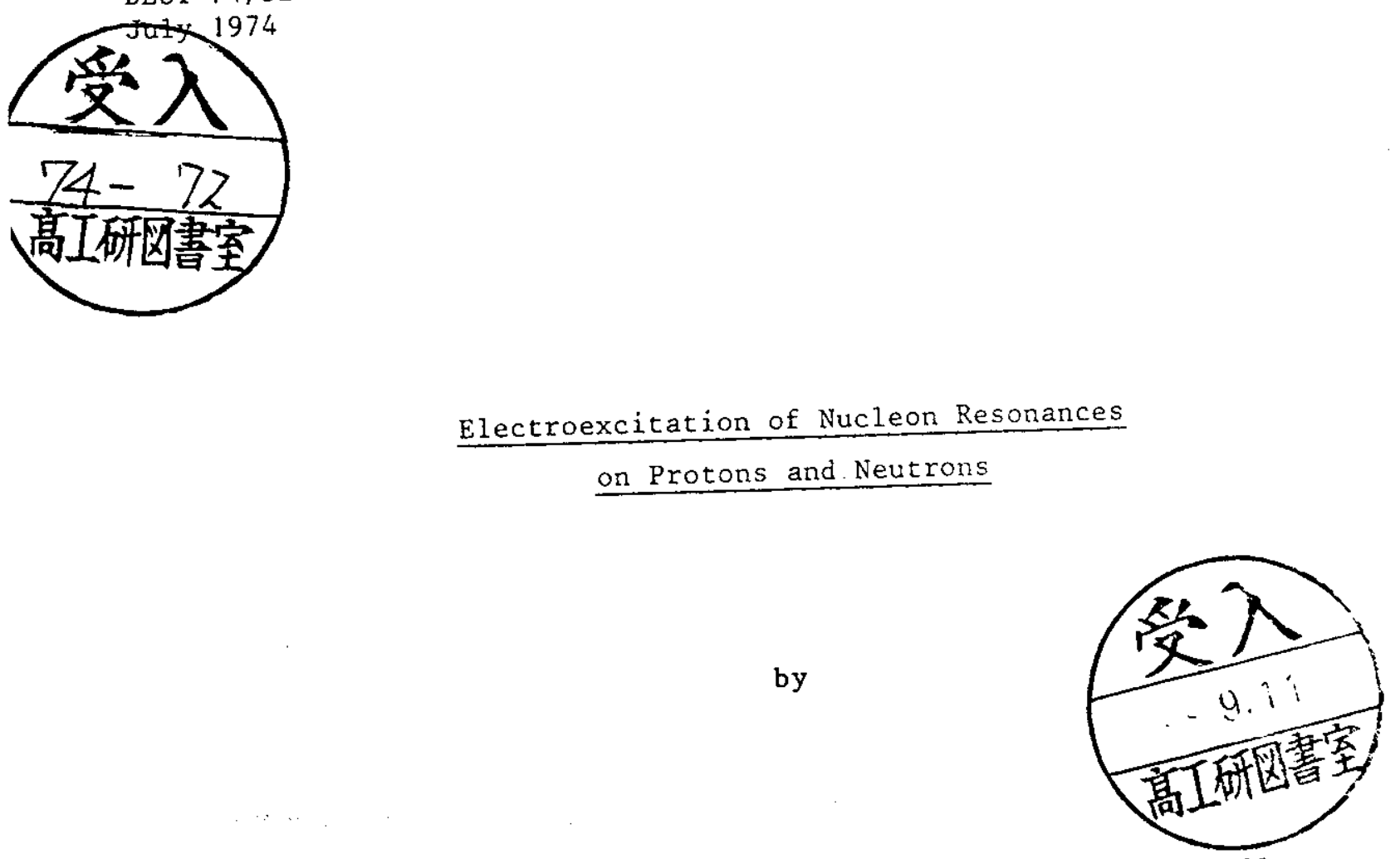

M. Köbberling, J. Moritz, K.H. Schmidt, D. Wegener, D. Zeller Institut für Experimentelle Kermphysik der Universität und des Kernforschungszentmons Karlsmihe,

\title{
J. Bleckwenn
}

Deutsches Elektronen-Synchrotron DESY, Hamburg,

and

F.H. Heim1ich

Fakultät für Physik der Universität, Freiburg 
EIFCTROEXOTTATTON OF NUCLEON RESONANCES

ON PROTONS AND NEUTRONS

" Krbberling, J. Moritz, K.H. Schmidt, D. Wegener*, D. Zeller

Tnstitut für Experimentelle Kernphysik der Universität und des Kernforschungszentrums Karlsruhe

J. Bleckwenn

Deutsches Elektronensynchrotron DESY, Hamburg

E.H. Heimlich

Fakultat für Physik der Universität, Freiburg

\section{ABSTRACT}

New data for the total absorption cross section of virtual photons on protons and deuterons are used to determine the excitation cross-section of the three dominant nucleon resonances at $W=1236 \mathrm{MeV}, W=1520 \mathrm{MeV}$ and $\mathrm{W}=1685 \mathrm{MeV}$. The $q^{2}$-dependence of the excitation on protons and of the ratio for the excitation on deuterons and protons is given. The resuits are compared with the predictions of the symmetric quark model.

* Present address: CERN, Geneva 


\section{INTRODUCTION}

The absoprtion cross section of virtual photons on protons and neutrons for four-momentum transfers $0.1(\mathrm{GeV} / \mathrm{c})^{2} \leq \mathrm{a}^{2}$ $\leq 1.5(\mathrm{GeV} / \mathrm{C})^{2}$ and invariant masses $\mathrm{W} \leq 1.85 \mathrm{GeV}$ has been determined by the Karlsruhe-DESY collaboration. The experiment has been performed with a wire spark chamber spectrometer, using a cerenkov counter and a shower counter for particle identification. The details of the apparatus have been described in former publications ${ }^{1,2,3}$. The measured cross section, the corrections and the error sources are given in tabulated form in a previous paper ${ }^{4}$. In the present paper the contribution of the dominant nucleon resonances and the nonresonant background to the measured and radiatively corrected cross section is determined.

\section{ANALYSIS PROCEDURE AND RESULTS}

The measured twofold differential cross section is connected to the absorption cross section $\sigma_{\text {tot }}$ by

$$
\sigma_{\text {tot }}=\sigma_{t}+\varepsilon \sigma_{1}=\frac{1}{\Gamma_{t}} \frac{d^{3} \sigma}{d \Omega d E}
$$

Where $\varepsilon$ is the degree of transverse polarization of the virtual photons, $\Gamma_{t}$ is the flux of virtual photons. $\sigma_{t}$ and $\sigma_{I}$ are the absorption cross sections of transverse respectively longitudinal polarized virtual photons.

The resonance cross sections are derived from the absorption cross section on protons and deuterons in two steps. By fit- 
ting of the resonance and the background contributions to the proton data the resonance parameters were determined. To get the resonance contribution from the deuteron cross section the Fermi motion was taken into account.

The resonance contributions to the absorption cross section were parametrized by Breit-Wigner formulas. For the $\Delta(1236)$ resonance a relativistic Breit-Wigner formula with mass dependent width was chosen ${ }^{3}$.

$$
\begin{aligned}
\sigma_{1236} & =\frac{\pi \alpha \vec{q}^{2}}{2 K W \cdot M} \frac{\Gamma(W)}{\left(W-M_{1}^{*}\right)^{2}+\Gamma^{2}(W) / 4} G_{M}^{* 2}\left(q^{2}\right) \\
\Gamma(W) & =\frac{0.128\left(0.85\left|\vec{p}_{\pi}^{*}\right| / m_{\pi}\right)^{3}}{1+\left(0.85\left|\vec{p}_{\pi}^{*}\right| / m_{\pi}\right)^{2}}
\end{aligned}
$$

where

$\begin{array}{ll}\mathrm{M}_{1}^{*} & \text { resonance mass } \\ \overrightarrow{\mathrm{q}} & \text { three momentum of the virtual photon } \\ \mathrm{W} & \text { invariant mass of the hadronic final state } \\ \mathrm{G}_{M}^{*}\left(\mathrm{q}^{2}\right) \quad \text { transition form factor } \\ \alpha & \text { fine structure constant } \\ \mathrm{K} & \text { equivalent photon energy } \\ \overrightarrow{\mathrm{p}}_{\pi}^{*} & \text { pion momentum in the CM system of the resonance } \\ \mathrm{m}_{\pi} & \text { pion mass }\end{array}$

The second and third nucleon resonance are described by nonrelativistic Breit-Wigner formulas 


$$
\begin{aligned}
& \sigma_{1520}=A_{2}\left(q^{2}\right) \frac{\Gamma(1520)}{\left(W-M_{2}^{*}\right)^{2}+\Gamma^{2}(1520) / 4} \\
& \sigma_{1685}=A_{3}\left(q^{2}\right) \frac{\Gamma(1685)}{\left(W-M_{3}^{*}\right)^{2}+\Gamma^{2}(1685) / 4}
\end{aligned}
$$

To fit the nonresonant background a polynomial was used

$$
\sigma_{n r e s}=\sum_{n=1}^{N} a_{n}\left(q^{2}\right)\left(w-w_{t h}\right)^{n-1 / 2}
$$

where the square root term $\left(W-W_{t h}\right)^{1 / 2}$ takes into account the behaviour of nonresonant pion production at the threshold $w_{t h} \cdot N=3$ gives a sufficient fit and has been used throughout the analysis. Values $N>3$ have been tried, but they do not give significant different results.

The fit to the proton data leads to the following set of resonance parameters

$$
\begin{aligned}
& M_{1}^{*}=(1.222 \pm 0.005) \mathrm{GeV} \\
& M_{2}^{*}=(1.51 \pm 0.008) \mathrm{GeV} \quad \Gamma(1520)=0.08 \mathrm{GeV} \\
& M_{3}^{*}=(1.685 \pm 0.014) \mathrm{GeV} \quad \Gamma(1685)=0.1 \mathrm{GeV}
\end{aligned}
$$

This result agrees with the analysis of Bloom et $a 1 .{ }^{5}$ at higher four momentum transfers. An example of the fit to the measured proton cross sections is given if fig. 1. In fig. 2 the resonance cross sections of the proton as a function of the four momentum transfer are given. The results are summarized in table 1 and table 2 . 
To compare the excitation cross section of resonances on bound state protons with that one on bound state neutrons we have foicied the cross section of the free proton with the momentum distributions of the nucleons bound in the deuteron

$$
\frac{d^{2} \sigma}{d \Omega_{3} d E_{3}}\left(E_{1}, E_{3}, \theta\right)=\int d^{3} \vec{p}\left\{w(\vec{p}) F(\vec{p}) \frac{d^{2} \sigma_{0}\left(E_{1}^{*}, F_{3}^{*}, \theta^{*}\right)}{d \Omega_{3}^{*} d E_{3}^{*}} \frac{d E_{3}^{*}}{d E_{3}} \frac{d \Omega_{3}^{*}}{d \Omega_{3}}\right\}
$$

where in the laboratory frame

$$
\begin{aligned}
& \vec{p} \quad \text { momentum of the target nucleon } \\
& E_{1} \quad \text { energy of the primary electron } \\
& E_{3} \quad \text { energy of the scattered electron } \\
& \theta \quad \text { scattering angle of the electron }
\end{aligned}
$$

The kinematic variables measured in the rest system of the target nucleons are characterized by an asterisk. $w(\vec{p})$ is the momentum aistribution of the target-nucleons. It is calculated from the Fourier transform of the Hulthen wave function ( $5 \%$ d-state contribution). By the term $F(\vec{p})$ we take into account the influence of the movement of the target nucleons to the flux of the virtual photons. To solve the integral equation (6) the following representation of the twofold differential cross section is used:

$$
\frac{d^{2} \sigma}{d \Omega_{3} d^{\prime} E_{3}}=\sigma_{M o t t}\left\{w_{2}\left(q^{2}, \nu\right)+2 t g^{2}(\theta / 2) w_{1}\left(q^{2}, \nu\right)\right\} \equiv \sigma_{\operatorname{Mott}} f\left(\nu, q^{2}, \theta\right)
$$

Merging of (7) into (6) results in 


$$
\begin{aligned}
\frac{d^{2} \sigma}{d \Omega_{3} d E_{3}}\left(E_{1}, E_{3}, \theta\right) & =\sigma_{M o t t}\left(E_{1}, E_{3}, \theta\right) r d^{3} \vec{p}\left\{w(\vec{p}) f\left(\nu^{*}, q^{2}, \theta^{*}\right)\right. \\
& \left.\left(1-p_{x} \cdot \sin \theta-p_{z} \cos \theta\right)^{2}\right\} \\
v^{*} & =E_{1}^{*}-E_{3}^{*} \\
E_{1}^{*} & =E_{1}\left(1-p_{z}\right) \\
E_{3}^{*} & =E_{3}\left(1-p_{x} \cdot \sin \theta-p_{z} \cos \theta\right)
\end{aligned}
$$

The direction of the primary electron beam is taken as $\mathrm{z}$ axis, while the $x$-axis is placed in the electron scattering plane ( $h=c=M=1$ ).

From formula (8) follows that the folding procedure consists in a superposition of cross sections at constant $q^{2}$ and different $v^{*}$. At small electron scattering angles an energy interval proportional to $v$ contributes to the broadening, therefore the resonances with high masses are broadened strongest. The function $f\left(v^{*}, q^{2}, \theta^{*}\right)$ in formula ( 8 ) was determined by interpolation from the experimental values given in reference 4 .

This folding procedure has been applied separately to each of the resonant contributions determined from the proton cross sections. The folded resonance contributions and the Fermi broadened background

$$
\sigma_{D}=a_{i} \sigma_{f}(1236)+a_{2} \sigma_{f}(1520)+a_{3} \sigma_{f}(1680)+a_{4} \sigma_{f} \text { (nres) }
$$

have been fitted to the measured deuteron cross sections. Fig. 3 shows an example of a fit for a deuterium target. 
The constants $a_{i}, \ldots, a_{4}$ have been determined by the fit. The ratio of the single resonance term to the appropriate proton term in the maximum of the resonance measures the resonance excitation on neutrons. Corrections due to shadowing and the Pauli-principle are less than $6 \%$ and decrease with increasing four momentum transfer. Therefore no corrections of this kind have been applied to the data. Fif. 4 shows the ratio of resonance excitation on deuterons and protons as a function of the four momentum transfer in the region of the three nucleon resonances. These results are given in table 3.

\section{DISCUSSTON OF RHE RESULTS}

The excitation of the $\Delta(1236)$-resonance on protons and neutrons is characterized by the transition form factor $G_{M}^{*}\left(q^{2}\right)$ of formula (1). The results of the present analysis are given in fig. 5. Since only the isovector amplitude contributes to this transition form factor, the excitation of the $\Delta(1236)$-resonance on protons and neutrons should be equal. This result is in agreement with a former experiment which was performed in a smaller $a^{2}$-region ${ }^{6}$.

The prediction of the dispersion relation model of Gutbrod and Simon ${ }^{14}$ included in fig. 5, fits the experimental data. A dipole like formula has been fitted to the transition form factor:

$$
G_{M}^{*}\left(q^{2}\right)=\frac{\mu^{*}}{\left(1+q^{2} / 0.51\right)^{2}}
$$

where $\mathrm{q}^{2}$ is measured in $(\mathrm{GeV} / \mathrm{c})^{2}$. The transition moment 


$$
\mu^{*}=3.54 \pm 0.4
$$

determined from the $f i t$ is in agreement with results from photoproduction ${ }^{\text {? }}$.

Theoretical results for the excitation of higher nucleon resonances have been given by Ravnda ${ }^{8}$ and . Iipes ${ }^{9}$ in a relativistic version of the symmetric quark model. In figs. 6 and 7 the theoretical prediction and the experimental results for the proton target are compared. The $\mathrm{a}^{2}$ dependence and the strength of the measured cross section deviate from the predictions of the two models.

As fig. 6 shows, the differences between the Ravndal-model and the data for the excitation cross section of the $2^{\text {nd }}$-resonance is due to the large longitudinal cross section predicted by this model. Since the excitation of the $\mathrm{S}_{11}$ (1535)resonance is described satisfactorily by the Iipes version of the symmetric quark mode 10,11 one has to conclude that the theory of Lipes and the experiment differ mainly for the $D_{13}$ (1512)-resonance contribution.

The maximum of the resonance in the region of the $3^{\text {rd }}$-resonance is shifted to higher invariant masses with increasing four momentum transfer ${ }^{4}$. This trend is in agreement with the quark model predictions and supports the calculations which determine the contributions of the different nucleon resonances to the measured cross section. But fig. 7 demonstrates that in the detailed shape of the $q^{2}$-dependence and the absolute height of the cross section theory and experiment disagree. From this follows that the symmetric quark model, which is successful in photoproduction ${ }^{12}$ is only of limited predictive power in electroproduction. This is most probable due to the fact that the $\mathrm{q}^{2}$-dependence of the cross section is strongly affected by the quark wave function chosen. 
Close et al. ${ }^{13}$ have derived upper and lower limits for the ratio $\sigma_{N} / \sigma_{H}$ of the excitation cross section of nucleon resonances on neutrons and protons: which can be used to determine the ratio $\sigma_{D} / \sigma_{H}$ of the cross sections for resonance excitation on deuterons and protons. They have taken into account the difference between, constituent and current quarks by classifying the nucleon resonances according to $\mathrm{SU}(6)_{\mathrm{W}}$ in the constituent basis. Furthermore they used the most general form for the current which is compatible with it be-

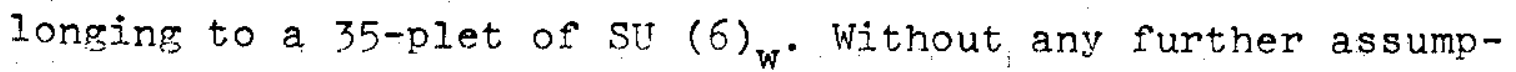
tions on the quark wave function upper and lower limits for the ratio $\sigma_{D} / \sigma_{H}$ of the different nucleon resonances can be derived from their calculations.

In the region of the $2^{\text {nd }}$ resonance the limits are given by $n \leq \sigma_{D} / \sigma_{H} \leq 2$ dependent on the contribution of the known three nucleon resonances in this mass interval. As shown by fig. $4 b$ this prediction is compatible with the results of the present analysis.

In the region of the $3^{\text {rd }}$ resonance, the model predicts for the $S_{31}(1650)$ and the $D_{33}$ (1670) resonance a ratio $\sigma_{D} / \sigma_{H}$ $=2$. The $D_{15}$ (1670) resonance should not be excited on protons and for the $F_{15}$ (1688) resonance the limit for the ratio is $0 \leq \sigma_{D} / \sigma_{H} \leq 4 / 9$. The results of fig. $4 \mathrm{c}$ are compatible with this model either if the $S_{31}$ (1650) and the $D_{33}$ (1670) resonance dominate the excitation cross section or if the $D_{15}(1670)$ and the $F_{15}$ (1688) resonance are excited with equal strength.

In conclusion it follows that the general results of the quark model are in good agreement with the experimental data, while specific theoretical predictions, which depend sensitevely on the wave function chosen, differ from the experimental results. 
ACKNOWIEDGEMENT

The help of Ing. H. Sindt during all stages of the experiment is acknowledged. This work has been supported by the Bundesministerium für Forschung und Technologie. 


\section{REFERENCES}

1. J. Moritz, K.H. Schmidt, D. Wegener, J. Bleckwenn,

E. Engels $j r$.

Nucl. Phys. B41 (1972), 336

2. J. Bleckwenn, H. Klein, J. Moritz, K.H. Schmidt,

D. Wegener

Nuc I. Phys. B33 (1971), 475

3. S. Galster, G. Hartwig, H. Klein, J. Moritz, K.H. Schmidt,

N. Schmidt-Parzefall, D. Wegener, J. Bleckwenn

Phys. Rev. D5 (1972), 519

4. M. Köbberling, J. Moritz, K.H. Schmidt, D. Wegener,

D. Zeller, J. Bleckwenn, F.H. Heimlich

Kernforschungszentrum KarIsruhe, KFK-Bericht 1822 (1973)

5. W.K.H. Panofsky

$14^{\text {th }}$ International Conference on Figh Energy Physics, Vienna 1968, p. 23

E. Bloom, D. Coward, H. De Staebler, J. Drees, J. Litt,

G. Miller, L. Mo, R.E. Taylor, M. Breidenbach,

J.I. Friedman, H.W. Kendall, S. Loken

Paper presented to the $14^{\text {th }}$ International Conference on High Energy Physics, Vienna 1968

M. Breidenbach

1970 PhD thesis Livs-MIT 2098-635 PhD thesis, IIT

6. J. Bleckwenn, J. Noritz, K.H. Schmidt, D. Wegener Phys. Lett. 38B (1972), 265 
7. G. Ebel, A. Milliensiefen, H. Pilkuhn, F. Steiner,

D. Wegener, M. Gourdin, C. Michael, J.I. Peterson,

I1. Roos, B.R. Martin, G. Oades, J.J. De Swart

Nucl. Phys. B33 (1971), 317

8. R.P. Feynman, M. Kislinger, F. Ravndal

Phys. Rev. D3 (1971), 2706

F. Ravndal

Phys. Rev. D4 (1971), 1466

9. R.G. Lipes

Phys. Rev. D5 (1972), 2849

1n. P.S. Kummer, E. Ashburner, F. Foster, G. Hughes,

R. Siddle, J. Allison, B. Dickinson, E. Evangelides,

M. Ibbotson, R.S. Lawson, R.S. Meaburn, H.E. Montgomery,

W.J. Shuttleworth

Phys. Rev. Lett. $30(1973), 873$

11. U. Beck, K.H. Becks, V. Burkert, J. Drees, G. Knop,

H. Kolanoski, M. Leenen, K. Moser, Ch. Nietzel,

J. Pysler, K. Rith, R. Sauerwein, E. Schlösser,

H.E. Stier

Bonn preprint, P I B 1-217 (August 1973)

12. J.K. Walker

Proc. of Internation Symposium on Electron and Photon

Interactions at High Energies, Liverpool 1969

13. F.E. Close, H. Osborn, A.M. Thomson

CERN preprint 1818 (February 1974)

14. F. Gutbrod, D. Simon

Nuovo Cimento 51A (1967), 602 


\section{TABLE 1}

Transition form factor $G_{M}^{*}\left(q^{2}\right)$ for the excitation of the $\Delta(1236)$-resonance on protons and neutrons

\begin{tabular}{l|c|c}
$q^{2}(\mathrm{GeV} / \mathrm{c})^{2}$ & $\mathrm{G}_{\mathrm{Mp}}^{*}$ & $\mathrm{G}_{\mathrm{Mn}}^{*}$ \\
\hline 0.15 & $2.15 \pm 0.13$ & \\
0.20 & $1.79 \pm 0.12$ & $1.76 \pm 0.15$ \\
0.30 & $1.38 \pm 0.04$ & $1.28 \pm 0.10$ \\
0.40 & $1.10 \pm 0.08$ & $1.02 \pm 0.08$ \\
0.50 & $0.92 \pm 0.03$ & $0.91 \pm 0.04$ \\
0.70 & $0.64 \pm 0.02$ & $0.60 \pm 0.03$ \\
1.0 & $0.39 \pm 0.02$ & $0.36 \pm 0.03$ \\
1.2 & $0.31 \pm 0.01$ & $0.31 \pm 0.02$ \\
1.5 & $0.22 \pm 0.01$ & $0.21 \pm 0.02$
\end{tabular}

TABIE: $2 \mathbf{a}$

Excitation cross section of the $\Delta(1236)$-resonance on protons

\begin{tabular}{l|r}
$q^{2}(\mathrm{GeV} / \mathrm{c})^{2}$ & $\sigma_{t}+\varepsilon \sigma_{1}|\mu \mathrm{b}|$ \\
\hline 0.15 & $690 \pm 100$ \\
0.20 & $580 \pm 80$ \\
0.30 & $460 \pm 30$ \\
0.40 & $370 \pm 40$ \\
0.50 & $315 \pm 15$ \\
0.70 & $210 \pm 15$ \\
1.0 & $110 \pm 10$ \\
1.2 & $85 \pm 5$ \\
1.5 & $50 \pm 5$
\end{tabular}




\section{TABLE 2b}

Excitation cross section of the $2^{\text {nd }}$ nucleon resonance on protons

\begin{tabular}{c|r}
$a^{2} \mid(G e V / c)^{2} !$ & $\sigma_{t}+\varepsilon \sigma_{1} \mid \mu b !$ \\
\hline 0.12 & $116 \pm 30$ \\
0.15 & $126 \pm 20$ \\
0.24 & $110 \pm 24$ \\
0.34 & $94 \pm 16$ \\
0.39 & $84 \pm 7$ \\
0.57 & $59 \pm 7$ \\
0.84 & $44 \pm 6$ \\
1.04 & $31 \pm 5$ \\
1.32 & $22 \pm 3$
\end{tabular}

TABLE 2C

Excitation cross section of the $3^{\text {rd }}$ nucleon resonance on protons

\begin{tabular}{c|c}
$\mathrm{q}^{2}\left|(\mathrm{GeV} / \mathrm{c})^{2}\right|$ & $\sigma_{\mathrm{t}}+\varepsilon \sigma_{1}|\mu \mathrm{b}|$ \\
\hline 0.21 & $68 \pm 20$ \\
0.30 & $75 \pm 16$ \\
0.33 & $43 \pm 17$ \\
0.48 & $37 \pm 7$ \\
0.74 & $35 \pm 15$ \\
0.93 & $32 \pm 6$ \\
1.20 & $24 \pm 9$
\end{tabular}




\section{TABIE $3 a$}

Ratio $\sigma_{D} / \sigma_{p}$ of cross sections for the excitation of the $\Delta(1236)$-resonance on deuterons and protons

\begin{tabular}{c|c}
$q^{2}\left|(G e v / c)^{2}\right|$ & $\sigma_{D} / \sigma_{p}$ \\
\hline 0.20 & $1.99 \pm 0.1$ \\
0.30 & $1.82 \pm 0.1$ \\
0.40 & $1.87 \pm 0.05$ \\
0.50 & $1.96 \pm 0.05$ \\
0.70 & $1.88 \pm 0.07$ \\
1.0 & $1.86 \pm 0.08$ \\
1.2 & $2.02 \pm 0.1$ \\
1.5 & $1.93 \pm 0.05$
\end{tabular}

PABIF $3 B$

Ratio $\sigma_{n} / \sigma_{p}$ of cross sections for the excitation of the $2^{\text {nd }}$ resonance on deuterons and protons

\begin{tabular}{c|c}
$a^{2}\left|(\mathrm{GeV} / \mathrm{c})^{2}\right|$ & $\sigma_{\mathrm{D}} / \sigma_{\mathrm{p}}$ \\
\hline 0.15 & $2.00 \pm 0.15$ \\
0.24 & $1.85 \pm 0.35$ \\
0.34 & $1.72 \pm 0.12$ \\
0.39 & $1.48 \pm 0.3$ \\
0.57 & $1.45 \pm 0.5$ \\
0.84 & $1.4 \pm 0.3$ \\
1.04 & $1.35 \pm 0.35$
\end{tabular}


TABIE $3 C$

Ratio $\sigma_{D} / \sigma_{p}$ of cross sections for the excitation of the $3^{\text {rd }}$ resonance on deuterons and protons

\begin{tabular}{c|c}
$\sigma^{2} !\left(G e^{\top / c}\right)^{2} \mid$ & $\sigma_{D} / \sigma_{p}$ \\
\hline 0.21 & $2.55 \pm 0.6$ \\
0.30 & $2.05 \pm 0.2$ \\
0.33 & $2.15 \pm 0.2$ \\
0.48 & $1.45 \pm 0.45$ \\
0.74 & $1.9 \pm 0.5$ \\
0.93 & $2.1 \pm 0.3$
\end{tabular}


Fig. 1: Separation of resonant and nonresonant contribution of a measured electron spectrum using a proton target.

Fig. 2: Cross section for the excitation of the $\Delta(1236)$-resonance, the $2^{\text {nd }}$ resonance and the $3^{\text {rd }}$ resonance as a function of the four momentum transfer $q^{2}$.

Fig. 3: Contribution of resonant and nonresonant processes to the measured electron spectrum for deuteron targets.

Fig. 4: Ratio of the cross sections for the resonance excitation on deuterons and protons as a function of the four momentum transfer.

Fig. 5: Transition form factor $G_{M}^{*}\left(a^{2}\right)$ as a function of the four momentum transfer. The solid line is the prediction of Gutbrod and Simon ${ }^{14}$.

Fig. 6: Cross section for the excitation of resonances on protons in the region of the $2^{\text {nd }}$ resonance as a function of the four momentum transfer. For comparison the predictions of Ravndal ${ }^{8}(-)$ and Lipes 9 $(.-.-)$ are included
1: $\sigma_{t}$
2: $\sigma_{1}$
3: $\quad \sigma_{t}+\varepsilon \sigma_{1}$

Fig. 7: Cross section for the excitation of resonances on protons in the region of the $3^{\text {rd }}$ resonance as a function of the four momentum transfer. For compa- 
rison the theoretical predictions of Ravndal ${ }^{8}$ (-) and Lipes ${ }^{9}(.-.-)$ are included
1: $\sigma_{t}$
2: $\sigma_{1}$
$3: \quad \sigma_{t}+\varepsilon \sigma_{1}$ 


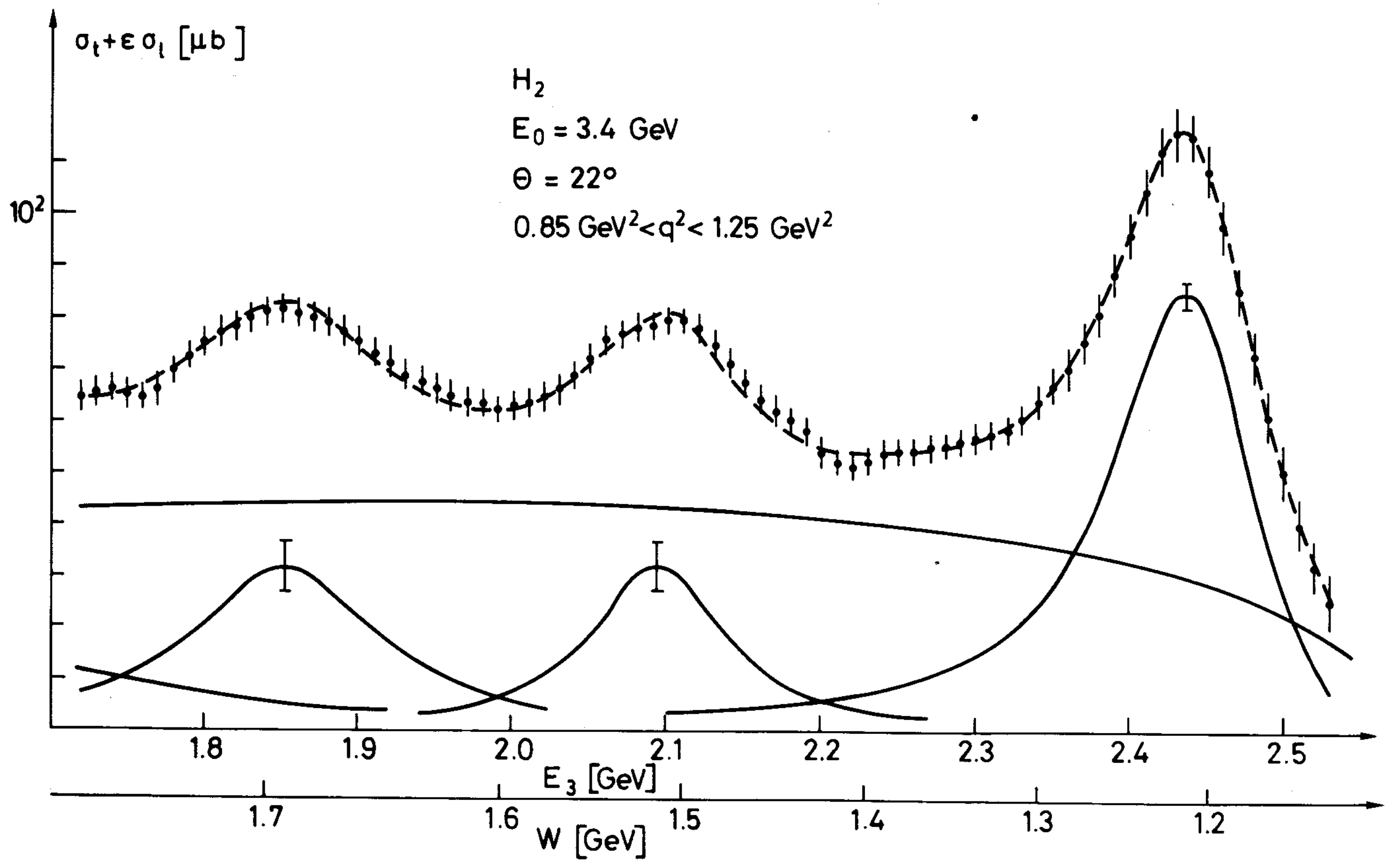

Fig. 1 


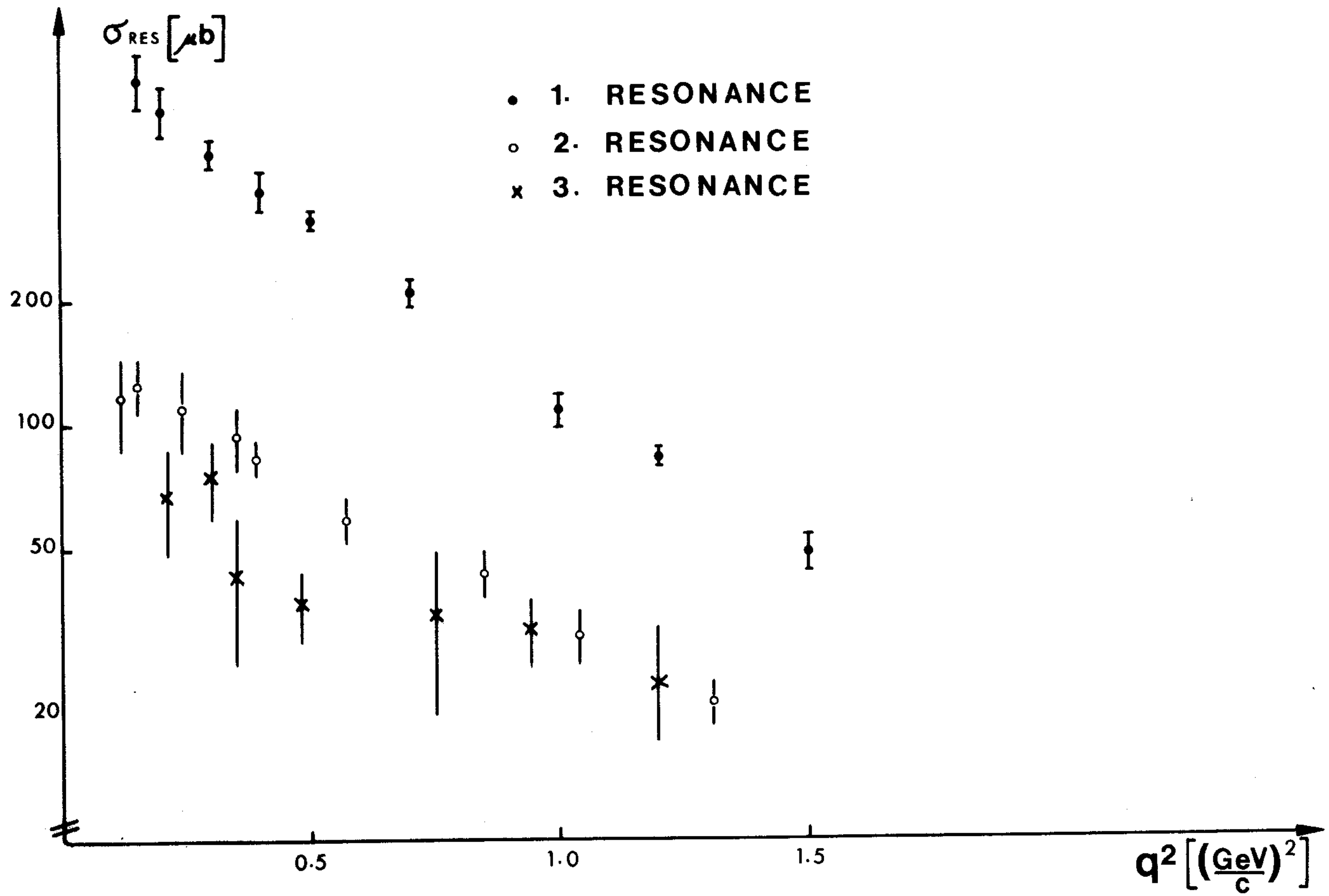

Fig. 2 


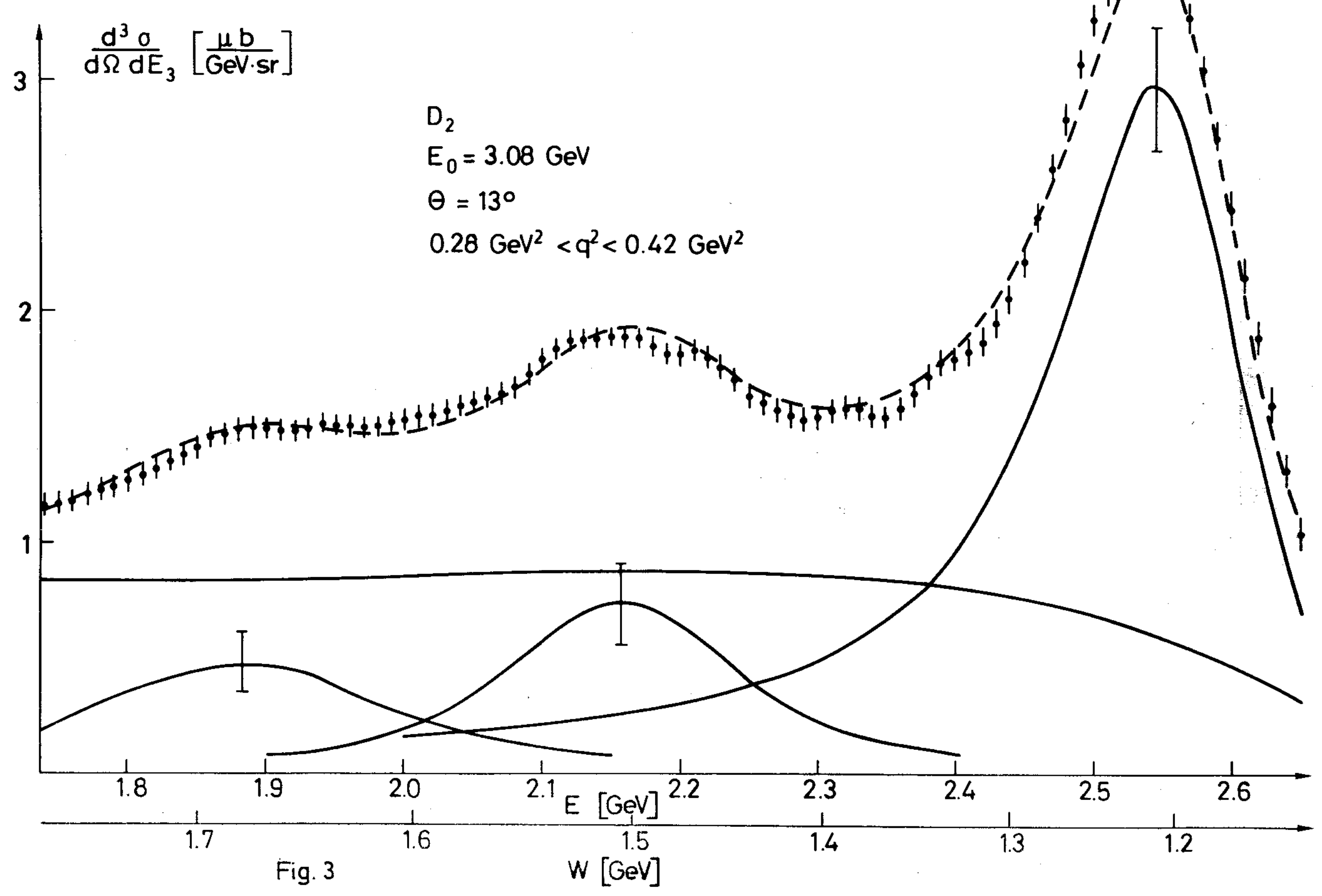



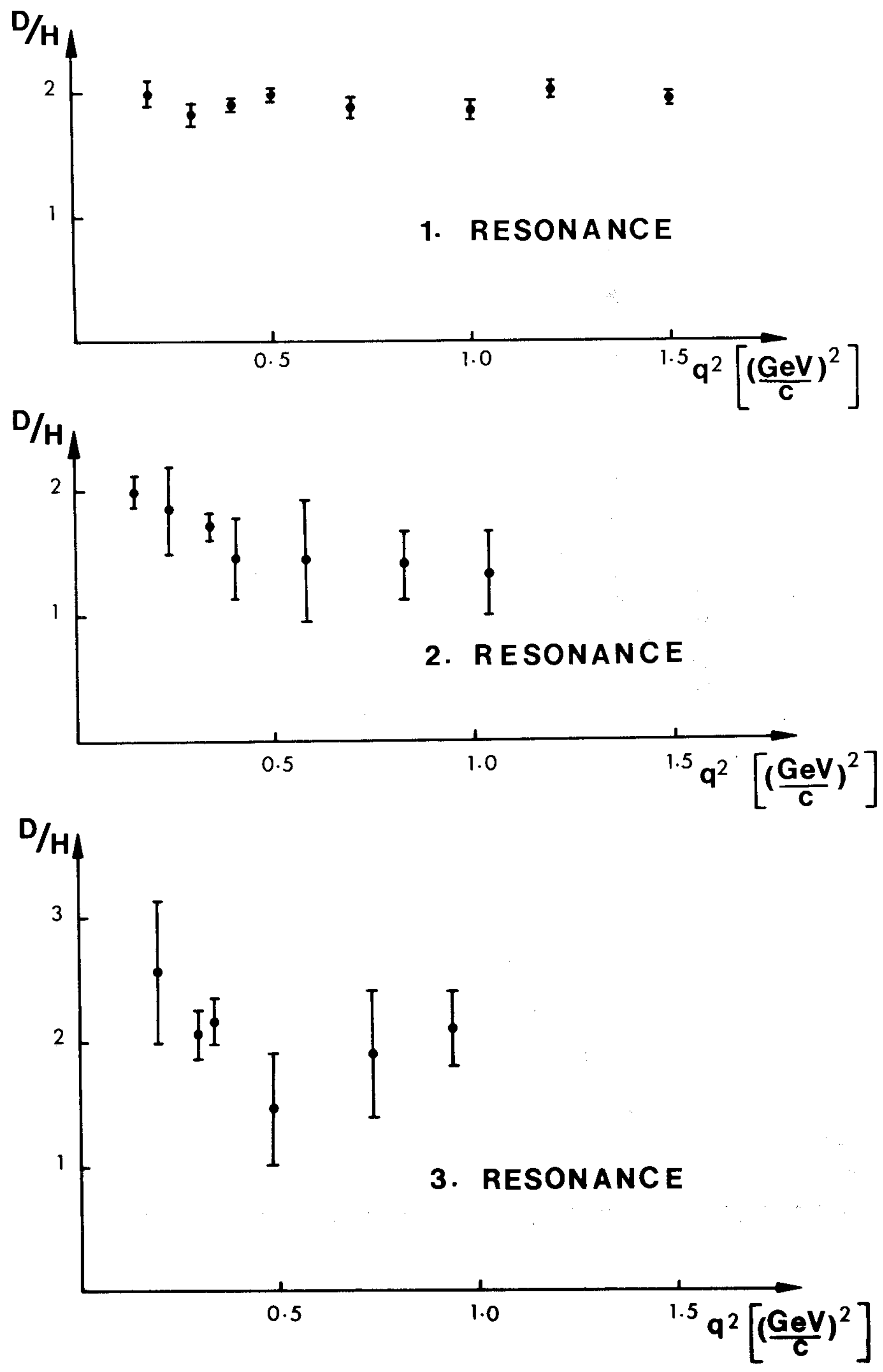

Fig. 4 


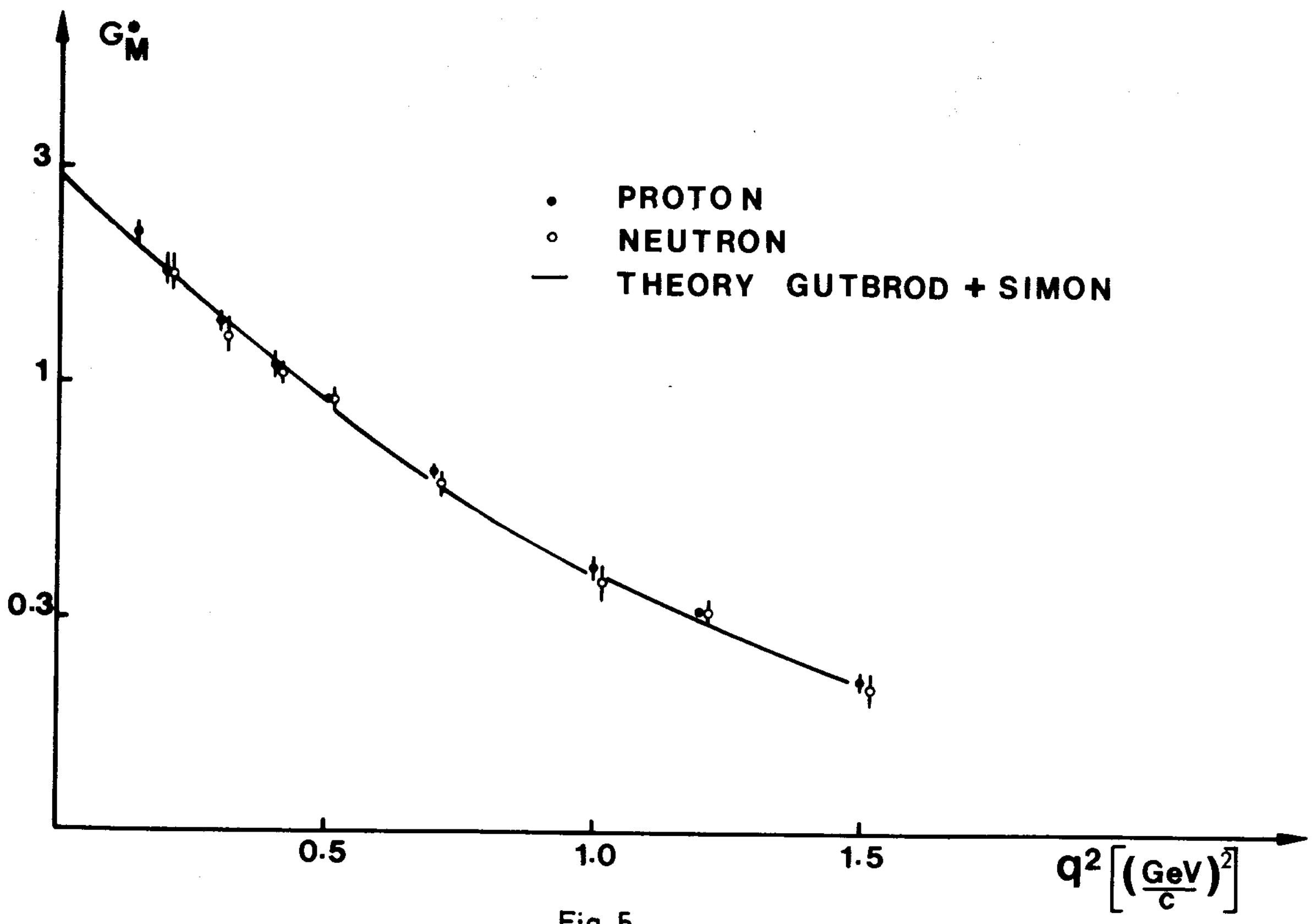

Fig. 5 


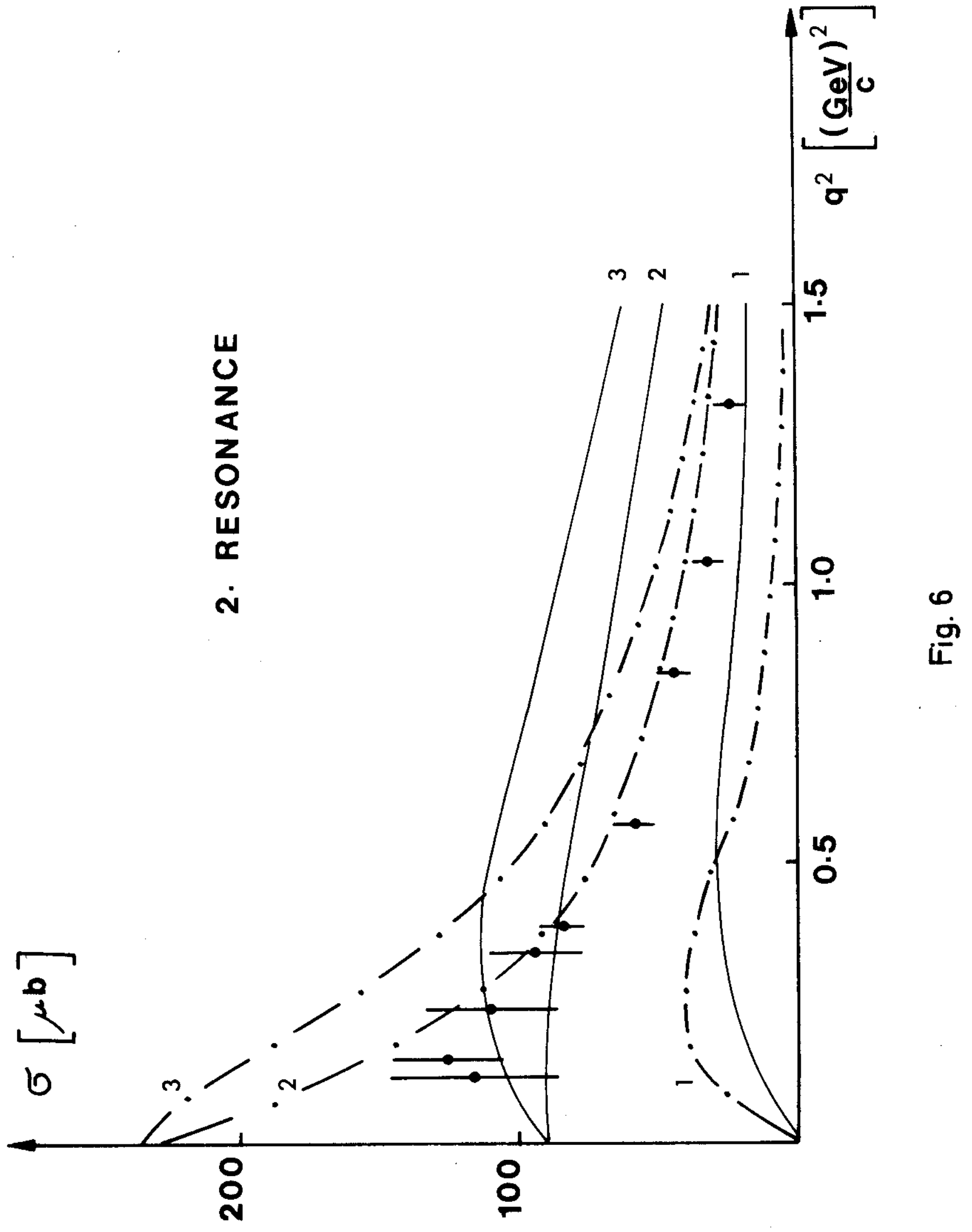




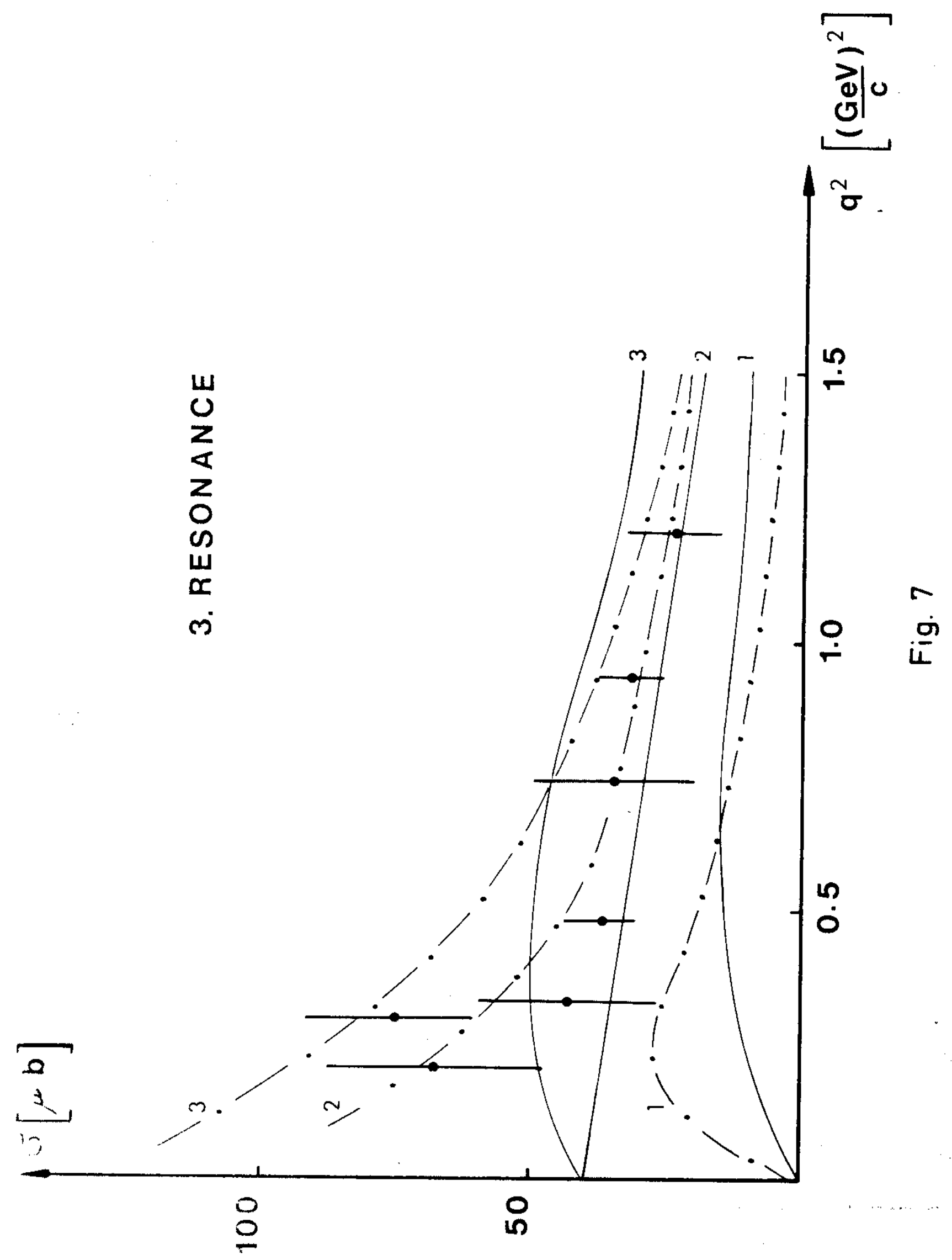

